\title{
Perioperative fluid management in pediatric patients
}

\author{
Mehmet Burak Eşkin ${ }^{1}$, Mehmet Emin Orhan ${ }^{1}$ \\ (1) University of Health Sciences, Gulhane Medical School, Anesthesiology and Reanimation, Ankara, Turkey
}

Date submitted:

Oct 15, 2018

Date accepted:

Dec 21, 2018

Online publication date:

June 15, 2019

\section{Corresponding Author: \\ Mehmet Burak Eşkin \\ University of Health \\ Sciences, Gulhane Medical \\ School, Anesthesiology and \\ Reanimation, Ankara, Turkey \\ burakeskin@hotmail.com}

Keywords: Pediatric Anesthesia, Fluid Management, hyperglycemia, hyponatremia.

\begin{abstract}
Perioperative fluid management is the application to prevent changes in the fluid compartments that may arise from surgery and anesthesia and the related electrolyte disorders. The aim is to provide adequate tissue perfusion in the perioperative period. Since even minor changes in fluid compartments in pediatric patients may affect physiological functions, it requires more importance compared to fluid management in adults. In this paper, it is aimed to update the knowledge about perioperative fluid treatment in pediatric patients, in accordance with the literature. According to recent studies, fasting times in the preoperative period should be reduced to 1 hour for clear fluids. Clinical protocols for oral fluids should be established in the perioperative period The Holiday-Segar formula (4/2/1), which was developed in 1957 according to caloric requirements in intraoperative fluid replacement, is still widely used. On the other hand, it is apparent that hyponatremia and hyperglycemia have a high risk of hypotonic fluid containing dextrose, according to this formula. It is not appropriate to use dextrose-containing fluids in children older than 4 weeks in the intraoperative period. If there is no contraindication in the postoperative period, enteral fluid intake must be started as soon as possible. In children with enteral feeding contraindicated, $5 \%$ glucose-containing fluids for children younger than 6 years of age, isotonic fluids containing $2 \%$ glucose with isotonic fluids for children older than 6 should be used as a postoperative maintenance fluid. Monitoring of fluid and electrolytes must be performed during the perioperative period of major surgical procedures.
\end{abstract}

The aim of perioperative fluid therapy is to provide adequate intravascular volume and cardiac output by applying intravenous solutions required to correct physiological functions that have been altered due to surgical stress and anesthetic agents and to maintain body homeostasis to provide oxygen to the tissues. In this way, the fluid deficiency is replaced, sufficient tissue perfusion is provided and the unwanted effects of anesthetics are tried to be removed.

In perioperative period, perioperative fluid deficits include hunger, gastrointestinal, renal or cutaneous losses, hemorrhage and third-space losses. These third-space losses are often due to extracellular losses caused by trauma and surgery.

\section{Perioperative Fluid Replacement in Pediatric Patients}

The maintenance fluid represents the fluid needed by the average person to maintain normal intracellular and extracellular volume over a 24 hour period. Therefore, the maintenance fluid must compensate the expected physiological losses such as breathing, sweating, and urination. For maintenance fluid replacement in pediatric patients; In 1957, Malcolm Holliday and William Segar calculated the metabolic needs of the pediatric patients older than 4 weeks during rest and activity by observing the energy needs of the children fed with different diets (glucose-water, human breast or cow's milk) and urinary sodium excretion. Metabolic calorie requirement and maintenance fluid requirement ( 1 calorie $=1 \mathrm{ml}$ water ) appear to be parallel to one another (1). According to this calculation, the amount of fluid to be given is $4 \mathrm{ml} / \mathrm{kg}$ for the first 10 kilograms of the child, $2 \mathrm{ml} /$ $\mathrm{kg}$ in addition to to $40 \mathrm{ml}$ and $1 \mathrm{ml} / \mathrm{kg}$ in addition to $60 \mathrm{ml}$, calculated with the formula $4 / 2 / 1$. The content of the fluid to be given is proposed to be close to the electrolyte composition of the human breast milk and cow milk, with the requirements of electrolyte as follows: $3 \mathrm{mEq} / 100 \mathrm{kcal}$ sodium (Na) per day and 2 $\mathrm{mEq} / 100 \mathrm{kcal}$ potassium (K) per day (1). This approach has been used in the perioperative fluid approach for more than a decade and is still widely used by most centers. However, most of the energy expenditure (80\%) occurs in the major metabolic organs (heart, liver, kidney, brain), which account for only $7 \%$ of the total body mass. Therefore, Holliday and Segar formula, which target the body mass, calculate the basal energy requirement greater than it actually is. Additionally, considering that pediatric patients are more inactive when compared to active, 
running and playing children, there are also insensible losses overcalculated. Regarding this, Oh et al. (2) In 1980, developed a new formula and proposed using $4 \mathrm{ml} / \mathrm{kg}$ for children between $3-10 \mathrm{~kg}, 20 \mathrm{ml}+$ weight $(\mathrm{kg}) \times 2 \mathrm{ml}$ for children between between $10-20 \mathrm{~kg}, 40 \mathrm{ml}+$ weight $(\mathrm{kg})$ for children over $20 \mathrm{~kg}$. Adelman et al. (3) calculated the body surface area according to [weight $(\mathrm{kg}) \times$ height $(\mathrm{cm})] / 3600$ in the year 2000 and suggested fluid replacement according to $1500 \mathrm{ml} / \mathrm{m} 2$ However, these formulas related to the amount of fluid to be given could not widely come into use to replace the stereotypical, memorized and customary Holiday-Segard formula. In perioperative fluid treatment, the amount of fluid given is as important as the amount of tonicity. In perioperative fluid management, adults are usually given isotonic solutions (such as lactated ringer or $0.9 \% \mathrm{NaCl}$ ) and for children, iso-osmolar dextrose- $\mathrm{NaCl}$ solutions (usually $5 \%$ or $10 \%$ dextrose, $0.225 \%$ or $0.45 \%$ hypotonic solutions such as $\mathrm{NaCl}$ ) are used and these solutions acts as hyptonic when dextrose in these solutions metabolized in vivo. The choice of isoosmolar dextrose- $\mathrm{NaCl}$ solutions is due to the fact that the water and salt content they contain is appropriate to the estimated baseline maintenance values. At the same time, the added dextrose to make the solution isoosmolar allows painless administration of the solution from a peripheral vein. In the last two decades, however, a debate has arisen that limits the use of these hypotonic maintenance fluids, and the discussions often address a series of iatrogenic hyponatremia cases reported in the literature. Concerns about intravenous (IV) hypotonic fluids have focused on potential neurological sequelae associated with severe hospital-induced hyponatremia $(4,5)$. Hyponatremia (serum sodium $<135 \mathrm{mEq} / \mathrm{L}$ ) is the most common electrolyte disorder in children, affecting approximately $25 \%$ of hospitalized children and $30 \%$ of children in the postoperative period (6). Hyponatremic encephalopathy is the most crucial risk of acute hyponatremia and may result in permanent neurological damage or death. Mortality in children with a blood sodium levels less than $129 \mathrm{mEq} / \mathrm{L}$ is around $8 \%$, while it is not clear what the actual incidence is, it is observed that $125 \mathrm{mEq}$ / $\mathrm{L}$ is associated with encephalopathy in $50 \%$ of the patients with serum fluid (7). The discussion of iatrogenic hyponatremia in pediatric patients has been most extensively examined in the postoperative pediatric population, a group that is at high risk and more accessible to research, albeit related to all hospitalized children. At least half of the cases documented with the diagnosis of hyponatremia in children occurred in the postoperative period, most of which occurred after uncomplicated surgeries (8). Although the given fluid is hypotonic and the hyponatremia develops due to its high volume, the effect of antidiuretic hormone (ADH) is also significant in the perioperative period. Stimulation of the antidiuretic hormone may be due to hemodynamic causes such as hypovolemia and hypotension, other factors that cause hemodynamic-independent non-osmotic ADH release include postoperative status, positive pressure ventilation, pain, nausea, vomiting and the use of narcotic medication (morphine, etc..) $(9,10)$. For this reason, positive pressure ventilation during general anesthesia in intraoperative period, narcotic analgesics used for pain, nausea, vomiting and postoperative analgesia in the postoperative period lead to an increase in $\mathrm{ADH}$ release. As a result of decreased diuresis effect of kidney due to ADH overstimulation, fluid retention and related dilutional hyponatremia increase the risk of hyponatremic encephalopathy in pediatric patients in perioperative period $(9,10)$.

Two methods have been emphasized to avoid hyponatremia in perioperative pediatric fluid management. First; it is suggested that the amount of fluid calculated with the present formula is too much and that the amount of hypotonic fluids to be given should be up to $60 \%$, instead of giving the whole of the calculated value $(8,10)$. This approach recommends the use of colloid or isotonic solutions for hypovolemia and persistent fluid loss. This leads to two separate fluid replacements. The second opinion is that $0.9 \% \mathrm{NaCl}$ should be used as maintenance fluid. In this application, however, it is put forth that the sodium concentration of sodium given to children will increase by about 5 times and hyperchloremic metabolic acidosis may be observed in some children. It has been suggested to use lactate ringer solution to prevent hyperchloremic metabolic acidosis (10). The increased electrolyte charge can be eliminated by introducing less isotonic maintenance fluid than currently recommended. In children at risk of hypoglycaemia, dextrose may be added to both isotonic and lactated ringer solution. However, the main problem of this method is that it enlarges the extracellular space in all cases and this may cause problems in some pediatric patients. Moreover, the free water required in this approach cannot be met. Another important point is that isotonic fluid delivery does not prevent postoperative hyponatremia. A decrease in the serum sodium levels were observed within the first 24-36 hours after surgery in patients who were given isotonic solutions in the perioperative period $(5,6)$. During this decrease, the patients developed more hypertonic urine compared to their serum. In the case, called desalination, extracellular fluid is increased due to the fluid given, natriuresis is triggered, yet patients are also under the effect of $A D H$. In case the patients were not affected by $\mathrm{ADH}$, they would produce a dilute, abundant urine, $\mathrm{ADH}$ causes free water retention and a hypertonic urine is formed as a result $(3,10,11)$.

\section{Preoperative Period}

Prevention of oral intake in children for a long time to minimize the risk of pulmonary aspiration which may have catastrophic consequences during perioperative period; causes harmful physiological and metabolic effects along with increased perioperative agitations $(11,12)$.

The incidence of hypoglycemia during induction of anesthesia is reported to be between $0 \%$ and $2.5 \%$. In most of the children identified with hypoglycemia, an average of 10 hours of fasting times are reported. Hypoglycemia was not observed in children who had drunk clear fluid up to 2 hours before surgery $(12,13)$. Until recently, it has been stated that the risk of pulmonary aspiration in children has an incidence of $0.07-0.1 \%$, the duration of fasting should be 2 hours for liquid foods, 4 hours for breast milk and formula, 6 hours for solid foods (14-18). In the latest APRICOT study, however, the risk of aspiration was found to be at the ratio of $9.3 \% / 10000=0.0093 \%(19)$. In healthy children, $1 \mathrm{ml} / \mathrm{kg}$ of these fluids were shown to have moved away from the stomach contents within 1 hour after the ingestion of clear fluids (20). Water discharges from the stomach within 30 minutes and other clear fluids leave the stomach almost completely within one hour. There is no difference observed in gastric volume or $\mathrm{pH}$ when clear fluids are given to children 1 or 2 hours before the operation. Clear glucose-containing fluids leave the stomach significantly faster (20-21). In the light of recent literature, it is suggested that children who will undergo surgery should receive clear fluids containing 3 $\mathrm{ml} / \mathrm{kg}$ glucose over the last 1 hour before surgery, according to their weight calculated. It is even emphasized that it would be practical to standardize $55 \mathrm{ml}$ for the ages between 1 and 
5 years, $140 \mathrm{ml}$ for $6-12$ years of age, and $250 \mathrm{ml}$ for over 12 years of age (20-22).

\section{Intraoperative Period}

Holliday and Segar protocol is still widely used as the most common formula to calculate fluid volume in the intravenous period $(1,23)$. The goal is to maintain the basal fluid electrolyte balance in a healthy child. Apart from special cases, perioperative maintenance fluids should be isotonic or near-isotonic (plasma-like tonicity). The use of hypotonic fluids is not recommended due to the risk of hyponatremic morbidity and mortality in the perioperative period, except in cases of free water loss (24). The rate of glucose in the perioperative fluid to be applied should not exceed $2 \%$ (25-26). In pediatric patients, fluids containing glucose as replacement fluids for unusual conditions (such as bleeding, vomiting, third-space losses) should not be used as they lead to hyperglycemia and hyponatremia $(24,26)$.

When iatrogenic hyponatremia occurs, iatrogenic hyperglycemia occurs too. This hyperglycemia, in addition to causing physiological and metabolic effects in the brain especially and other organs, increases the fluid reabsorption in the renal proximal tubules and causes osmotic diuresis which in return leads to an increase in the hypovolemia. Intraoperative initial fluid replacement should be performed with isotonic crystalloids. The superiority of different isotonic crystalloid solutions to one another has not been demonstrated $(8,27,28)$. In order to prevent hyperchloremic acidosis due to the use of $0.9 \% \mathrm{NaCl}$ in rapid and significant volume changes, it seems reasonable to use a more isotonic stable crystalloid with content similar to the plasma electrolytes closer to physiological $\mathrm{pH}(29,30)$. Colloids are an option for massive and acute replacement (albumin, gelatin or hydroxyethyl-starch). However, it is unclear even in non-pediatric patients whether colloids play a role in non-urgent volume replacement and which colloid is more effective in preventing the passage of fluid into the interstitial space and preventing associated complications. Probably the best approach is to avoid hypervolemia and hypovolemia (31).

In the same vein, there are publications showing that postoperative nausea and vomiting are observed less frequently in children who received liberal fluid $(30 \mathrm{ml} / \mathrm{kg} / \mathrm{hour}$ ) application in intraoperative period (32). The risk of hypoglycemia increases in newborns in the first 48 hours of life or in children fed with total parenteral nutrition However, for pediatric patients in none of these cases, it gives stress response to hunger and surgery, similar to adults, and often develops hyperglycemia, even if no dextrose is given in these children.

\section{Postoperative Period}

After small and uncomplicated surgeries, there is no need for postoperative fluid therapy unless oral fluid disorder is present. Unless there is a contraindication case, oral feeding should be started as soon as possible. In major surgeries or surgeries with no nutrition following operation, $5 \%$ glucose-containing isotonic fluids for children younger than 6 years of age, isotonic fluids containing $2 \%$ glucose with isotonic fluids for children older than 6 are recommended to use as a postoperative maintenance fluids. If necessary, it is recommended to perform isotonic fluid replacement alone and to maintain maintenance fluid replacement on the day after surgery (8). If a hypovolemic condition is suspected in the postoperative period, replacement with isotonic crystalloid solutions $(10-30 \mathrm{ml} / \mathrm{kg}$ ) or colloids $(10 \mathrm{ml} / \mathrm{kg})$ is recommended avoiding the use of hypotonic solutions (33).

\section{Monitoring Fluid Status}

In pediatric major surgeries where fluid and blood loss are expected, clinical evaluation of the perioperative fluid status of the patient should be performed (28). Since excessive and prolonged fluid and blood replacement is applied to these patients, blood hematocrit, hemoglobin, electrolyte and glucose levels should be monitored periodically in intraoperative and postoperative periods, and appropriate replacement should be carried out in accordance with electrolyte changes detected (33). In clinical conditions that may lead to hypoglycemia or hyperglycemia, perioperative glucose monitoring is recommended (34). In patients with headache, nausea, vomiting, irritability, altered level of consciousness, seizures, neurogenic pulmonary edema and apnea, all of which cause hyponatremia to be suspected, blood sodium level must be determined urgently $(20,46)$. The lack of reliable non-invasive monitoring of vascular volume to ensure an optimal fluid volume in pediatric patients is a major challenge (35).

As a result, despite current guidelines and publications, preoperative fasting and water intake times in children are much longer than recommended times due to anesthesiologists who are afraid of the risk of aspiration pneumonia and also more than half of the anesthesiologists still prefer hypotonic fluids because of their fear of hypoglycemia in younger children with prolonged surgery (10).

In the light of this information, the hydration status of pediatric patients should be evaluated with clinical examination in the preoperative period. The preoperative fasting period of pediatric patients should be determined as 1 hour for liquid foods and a clinical protocol should be established to this end. It should be remembered that there is a risk of hyponatremia, rather than hypoglycemia, in every stage of the perioperative period. Isotonic fluids should be preferred during intraoperative period and bleeding, fluid and electrolyte levels should be monitored in prolonged or major surgeries. In the postoperative period, oral intake should start as early as possible, and when oral intake is contraindicated, isotonic fluids containing $5 \%$ dextrose for children under 6 years of age and $2 \%$ for children over 6 years of age should be started to apply.

\section{Acknowledgement}

Manuscript was written by MBE and MEO. There is no financial assistance with this manuscript.

\section{Conflict of Interest}

The author did not declare anything to disclose regarding conflict of interest with respect to this manuscript.

\section{References}

1. Holliday MA, Segar WE. The maintenance need for water in parenteral fluid therapy. Pediatrics.1957;19(5): 823-832

2. Oh TH. Formulas for calculating fluid maintenance requirements. Anesthesiology. 1980; 53(4):351.

3. Adelman RD, Solhaugh MJ. Pathophysiology of body fluids and fluid transfer. In: Nelson's Textbook of Pediatrics, 16th ed. Edited by Behrman RE, Kliegman RM, Jenson HB. Philadelphia, PA: W.B. Saunders Company; 2000;(1) 189-224

4. Moritz ML, Ayus JC. Prevention of hospital-acquired hyponatremia: a case for using isotonic saline. Pediatrics 
2003; 111(2):227-230

5. Eulmesekian PG, Perez A, Minces PG, Bohn D. Hospital-acquired hyponatremia in postoperative pediatric patients: Prospective observational study. Pediatr Crit Care Med. 2010; 11(4): 479-483.

6. Holliday MA, Friedman AL, Segar WE, Chesney R, Finberg L. Acute hospital-induced hyponatremia in children: a physiologic approach. J Pediatr 2004; 145(5):584-587

7. Moritz ML, Ayus JC. New aspects in the pathogenesis, prevention, and treatment of hyponatremic encephalopathy in children. Pediatr Nephrol 2010; 25 (7) 1225-1238.

8. Moritz ML, Ayus JC. Intravenous fluid management for the acutely ill child. Curr Opin Pediatr 2011; 23(2): 186193.

9. Park KS, Yoo KY. Role of vasopressin in current anesthetic practice. Korean J Anesthesiol. 2017; 70(3):245257

10. Khan MF, Siddiqui KM, Asghar MA. Fluid choice during perioperative care in children: A survey of present-day proposing practice by anesthesiologists in a tertiary care hospital. Saudi J Anaesth. 2018; 12(1):42-45.

11. Denhardt N, Beck C, Huber D, et al. Optimized preoperative fasting times decrease ketone body concentration and stabilize mean arterial blood pressure during induction of anaesthesia in children younger than 36 months: a prospective observational cohort study. Pediatr Anesth. 2016; 26(8): 838-843.

12. Frykholm P, Schindler E, Sumpelmann R. Pre-operative fasting in children. A review of the existing guidelines and recent developments. Br J Anaesth. 2018; 120(3):469474

13. Paut O, Lacroix F. Recent developments in the perioperative fluid management for the paediatric patient. Curr Opin Anaesthesiol. 2006; 19(3):268-277.

14. Kelly $C$, Walker R. Perioperative pulmonary aspiration is infrequent and low risk in pediatric anesthetic practice. Pediatr Anesth. 2015; 25(1):36-43.

15. Tan Z, Lee S. Pulmonary aspiration under GA: a 13-year audit in a tertiary pediatric unit. Pediatr Anesth. 2016; 26(5):547-552.

16. American Society of Anesthesiologists Committee. Practice guidelines for preoperative fasting and the use of pharmacologic agents to reduce the risk of pulmonary aspiration: Application to healthy patients undergoing elective procedures: an updated report by the American Society of Anesthesiologists Committee on Standards and Practice Parameters. 2018; 114(3):495-511.

17. Busto-Aguirreurreta N, Munar-Bauza F, Fernández-Jurado $\mathrm{MI}$, et al. Perioperative fluid therapy in the pediatric patient. Recommendations. Rev Esp Anestesiol Reanim 2014; 61 Suppl 1:1-24.

18. López Muñoz AC, Busto Aguirreurreta N, Tomás Braulio J .Preoperative fasting guidelines: An update.. Rev Esp Anestesiol Reanim 2015; 62(3):145-156

19. Habre W, Disma N, Virag K, et al. Incidence of severe critical events in paediatric anaesthesia (APRICOT): a prospective multicentre observational study in 261 hospitals in Europe. Lancet. 2017; 5(5):412-425

20. Schmidt A, Buehler P, Seglias L, et al. Gastric pH and re- sidual volume after 1 and $2 \mathrm{~h}$ fasting time for clear fluids in children. Br J Anaesth. 2015; 114(3):477-482.

21. Bonner JJ, Vajjah P, Abduljalil K, et al. Does age affect gastric emptying time? A model-based meta-analysis of data from prematüre neonates through to adults. Biopharm Drug Dispos. 2015; 36(4):245-257.

22. Thomas M, Morrison C, Newton R, Schindler E.. Consensus statement on clear fluids fasting for elective pediatric general anesthesia. Paediatr Anaesth. 2018; 28(5):411-414.

23. Mandee S, Butmangkun W, Aroonpruksakul N, et al. Effects of a restrictive fluid regimen in pediatric patients undergoing major abdominal surgery. Paediatr Anaesth 2015; 25(5):530-537.

24. Neilson J, O'Neill F, Dawoud D, Crean P. Intravenous fluids in children and young people: Summary of NICE guidance. BMJ 2015; 9;351:h6388

25. Sümpelmann R, Mader T, Dennhardt N, Witt L, Eich $C$, Osthaus WA. A novel isotonic balanced electrolyte solution with $1 \%$ glucose for intraoperative fluid therapy in neonates: results of a prospective multicentre observational postauthorisation safety study (PASS). Paediatr Anaesth. 2011; 21(11):1114-1118.

26. Sumpelmann R, Becke K, Crean P, et al. German Scientific Working Group for Paediatric Anaesthesia European consensus statement for intraoperative fluid therapy in children.. Eur J Anaesthesiol. 2011; 28(9):637-639.

27. Moritz ML, Ayus JC. Prevention of hospital-acquired hyponatremia: do we have the answers? Pediatrics 2011; 128(5):980-983.

28. Bailey AG, McNaull PP, Jooste E, Tuchman JB. Perioperative crystalloid and colloid fluid management in children: where are we and how did we get here? Anesth Analg 2010 ;110(2): 375-390.

29. Sumpelmann R, Mader T, Dennhardt N, et al. A novel isotonic balanced electrolyte solution with $1 \%$ glucose for intraoperative fluid therapy in neonates: Results of a prospective multicentre observational postauthorisation safety study (PASS). Paediatr Anaesth 2010; 20(11): 977-981.

30. Long E, Duke T. Fluid resuscitation therapy for paediatric sepsis. J Paediatr Child Health 2016; 52(2):141-146.

31. O'Brien F, Walker IA. Fluid homeostasis in the neonate. Paediatr Anaesth 2014;24(1):49-59

32. Ashok V, Bala I, Bharti N, Jain D, Samujh R. Effects of intraoperative liberal fluid therapy on postoperative nausea and vomiting in children-A randomized controlled trial. Paediatr Anaesth. 2017; 27(8):810-815.

33. Murat I, Dubois MC. Perioperative fluid therapy in pediatrics Paediatr Anaesth 2008; 18(5):363-370.

34. Way C, Dhamrait R, Wade A, Walker I. Perioperative fluid therapy in children: A survey of current prescribing practice. Br J Anaesth 2006; 97(3):371-379.

35. Murat I, Humblot A, Girault L, Piana F. Neonatal fluid management. Best Pract Res Clin Anaesthesiol 2010; 24(3):365-374 